\title{
A circumferential rectal superficial neoplasm resected with endoscopic submucosal dissection using the pocket-creation method
}

\section{다)(1) $\odot$}

\author{
Authors \\ Konishi $^{1}$, Yuji Naito ${ }^{1}$, Mitsuo Kishimoto ${ }^{2}$, Takashi Ando ${ }^{3}$, Yoshito Itoh ${ }^{1}$ \\ Institutions \\ 1 Department of Molecular Gastroenterology and \\ Hepatology, Graduate School of Medical Science, Kyoto \\ Prefectural University of Medicine, Kyoto, Japan \\ 2 Department of Surgical Pathology, Graduate School of \\ Medical Science, Kyoto Prefectural University of \\ Medicine, Kyoto, Japan \\ 3 Ando Clinic, Kyoto, Japan
}

Rafiz Abdul Rani ${ }^{1}$, Naohisa Yoshida ${ }^{1}$, Takaaki Murakami ${ }^{1}$, Kiyoshi Ogiso ${ }^{1}$, Ryohei Hirose', Yutaka Inada ${ }^{1}$, Hideyuki

submitted 26.1.2017

accepted after revision $\quad 18.12 .2017$

\author{
Bibliography \\ DOI https://doi.org/10.1055/s-0044-101349 | \\ Endoscopy International Open 2018; 06: E484-E488 \\ (c) Georg Thieme Verlag KG Stuttgart · New York \\ ISSN 2364-3722
}

Corresponding author

Naohisa Yoshida, MD PhD, Department of Molecular

Gastroenterology and Hepatology, Kyoto Prefectural
University of Medicine, Graduate School of Medical Science, 465 Kajii-cho, Kawaramachi-Hirokoji, Kamigyo-ku, Kyoto 602-8566, Japan

Fax: $+81-75-2510710$

naohisa@koto.kpu-m.ac.jp

\section{ABSTRACT}

Endoscopic submucosal dissection (ESD) is recognized as an important technique in the nonsurgical management of early gastrointestinal carcinoma and it is continuously undergoing evolution in terms of technique and equipment. The pocket-creation method was recently developed for overcoming various difficulties such as large tumor size and severe fibrosis in ESD. Circumferential ESDs are rare and pose technical difficulties. We present a case of a circumferential rectal ESD using a pocket-creation method with two pockets. The associated planning, strategies, and outcome of the procedure are discussed.

\section{Introduction}

Endoscopic submucosal dissection (ESD) was first described for early colorectal carcinoma in the late 1990s [1] and it has evolved to become a leading technique in the nonsurgical management of early gastrointestinal carcinoma.

Colorectal ESD is challenging as the colorectal wall is thin by nature and the colon is long and winding. In addition, circumferential colorectal ESD increases the technical difficulty and has rarely been reported [2,3]. During ESD with a standard approach, the partially resected lesion sometimes blocks the lumen as well as the endoscopic view, resulting in diminished endoscope maneuverability and disorientation, therefore increasing procedural time $[2,3]$.

Recently, the pocket-creation method (PCM) has been developed to overcome various difficulties such as large tumor size and severe fibrosis in ESD [4]. A specific tapered hood is used for adequate traction in PCM and endoscopic operability is stable in the pocket. Thus, PCM allows for good traction to access the submucosal layer, even in anti-gravity positions and requires fewer patient position changes [4]. Additionally, the partially resected lesions do not block the lumen. We resected a circumferential superficial neoplasm using PCM with two pockets, which would normally be rather difficult, but was slightly less so using this technique.

\section{Case report}

A 35-year-old woman with a previous history of hemorrhoids presented with a positive fecal immunochemical test on health screening. She was asymptomatic from a normal physical examination and after blood investigations. Colonoscopy with white light examination showed a lesion circumferentially covering the low-perineal rectum with a longitudinal extension of $5 \mathrm{~cm}$ ( $\triangleright$ Fig. 1a). It was a lateral spreading tumor-granular type (LST-G). The lesion also partially extended into the anal canal. 

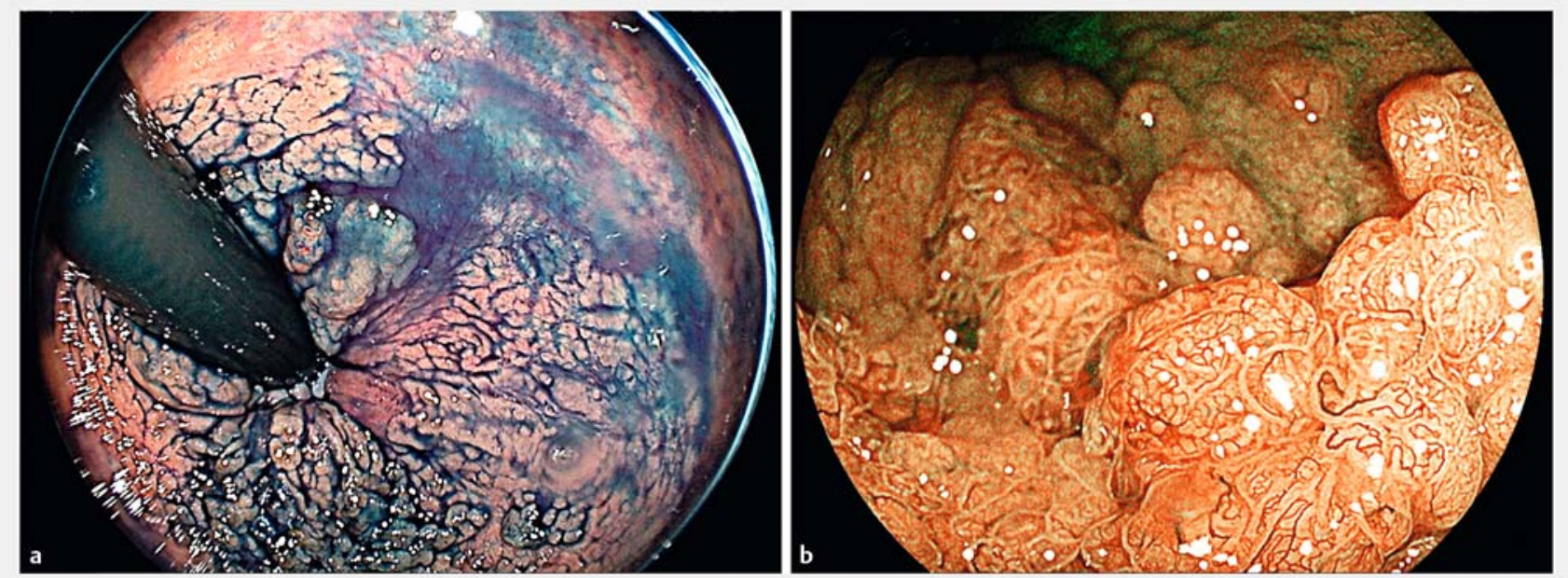

- Fig. 1 a A circumferential rectal lesion with indigo carmine spraying. b Blue laser imaging (BLI) magnification showed a mild irregular surface pattern and irregular vessel pattern with caliber change.

Magnified blue laser imaging (BLI, light source: LL-4450 light source, processor: VP-4450HD, Fujifilm Co., Tokyo, Japan) showed an irregular surface and a vessel pattern indicating Japanese NBI Expert Team (JNET) Classification Type 2B lesion ( $\bullet$ Fig.1b) $[5,6]$. A JNET Type 2B lesion indicates intramucosal cancer or high grade adenoma. For treatment, we decided against standard ESD and opted for PCM with two pockets because of the circumferential nature of the lesion.

We used a unique bowel preparation of $10 \mathrm{~mL}$ of sodium picosulfate 24 hours prior ESD. Then, from 4 hours prior ESD, 1.0 $\mathrm{L}$ of low-volume polyethylene glycol was administered, which has shown a good safety profile and efficacy [7]. For conscious sedation, the patient received a total of $14 \mathrm{mg}$ of intravenous midazolam (sedation), $15 \mathrm{mg}$ of pentazocine (pain-relief) and $10 \mathrm{mg}$ of hyoscine butylbromide (anti-spasmodic) throughout the procedure. We used the Fujifilm LASEREO system with ECL600ZP colonoscopy (diameter: $11.7 \mathrm{~mm}$, working channel: $3.2 \mathrm{~mm}$, length: $1300 \mathrm{~mm}$, Fujifilm Co., Tokyo, Japan) for diagnosis and ESD. The scope has a gradation of hardness for easier insertion and is more stable in ESD than previous scopes. In addition, a $3.2 \mathrm{~mm}$ channel is good for vacuuming. We also used a short ST hood (diameter: $16.5 \mathrm{~mm}, \mathrm{DH}-29 \mathrm{CR}$, Fujifilm Co.) for PCM [4]. For the injection liquid, in view of the anal canal invasion area, $10 \mathrm{~mL}$ of $1 \%$ Lidocaine and $0.5 \mathrm{mg}$ of epinephrine were added to the regular solution of $0.1 \mathrm{~mL}$ of indigo carmine and $20 \mathrm{~mL}$ of $0.4 \%$ hyaluronic acid (MucoUp, Boston Scientific Co., Tokyo, Japan). For the rectal area, we used a mixed liquid of $0.4 \%$ hyaluronic acid, indigo carmine, and epinephrine as described above. These were used to prevent pain and minor bleeding as well as to achieve long-lasting elevation. High flow injection needle (Impact flow: TOP Co. Tokyo, Japan) was used for injecting viscous hyaluronic acid solution.

In ESD, mucosal incision and dissection of the anal canal were performed first and were mainly conducted with the ClutchCutter (Fujifilm Co.) as its $3.5 \mathrm{~mm}$ jaw length allows coagulation of large hemorrhoidal vessels for a more efficient he-

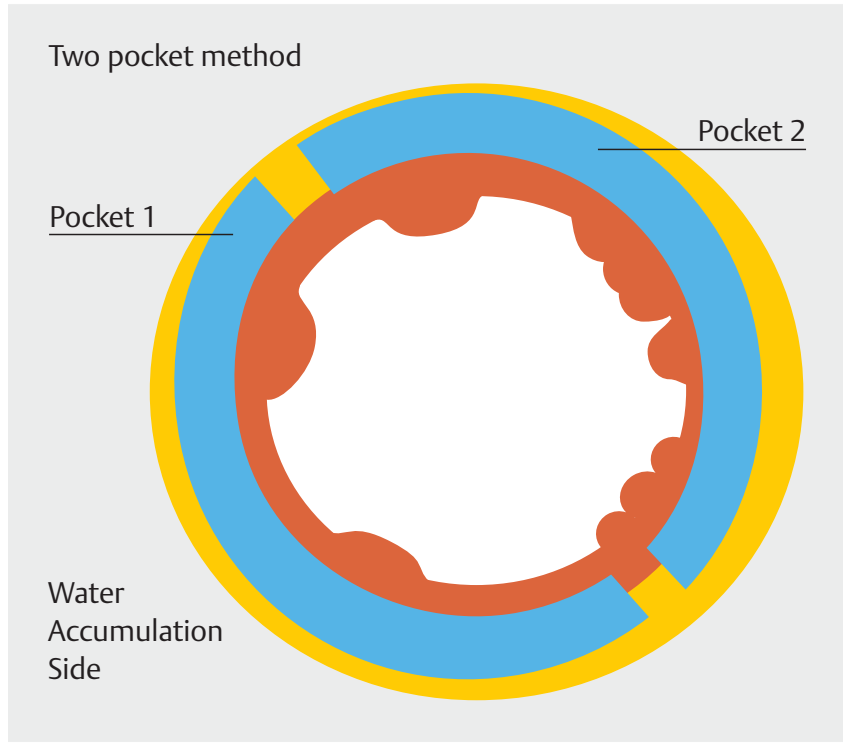

Fig. 2 Pocket-creation method showing the two half circumferential pockets. Pocket 1 was created first on the water accumulation side followed by pocket 2 . Subsequently, the mucosa on the oral side was incised and thereafter, we returned to the pockets sequentially to complete the dissection to the oral side like a tunnel. Finally, the submucosa in between the two pockets were resected and the en bloc specimen dissected. Red, rectal mucosa; blue, pockets in rectal submucosa; yellow, rectal muscle.

mostasis. Afterwards, the areas without thick vessels were resected with the FlushKnife BT-S $2.0 \mathrm{~mm}$ (Fujifilm Co.) which allows a quicker procedure. The first half circumferential pocket was created on the water accumulation side, then the second half circumferential pocket was made on the opposite side ( $\triangleright$ Fig.2). During dissection, the patient was repositioned appropriately to use gravity and obtain good traction. Several moderate perioperative hemorrhages occurred and they were 

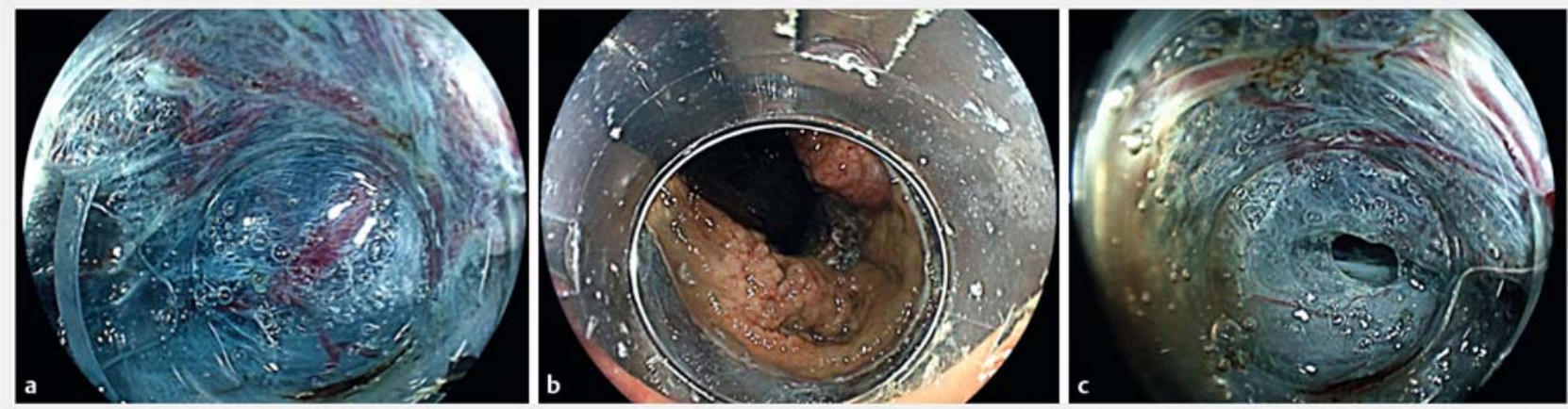

- Fig. 3 a Pocket formation requires thick vessels in the pocket to be dissected utilizing the ClutchCutter. b Pocket formation was followed by mucosal incision on the oral side. c subsequently, we returned to the pocket and continued dissection until reaching the oral side.

stopped with the ClutchCutter. After creating the two pockets, a mucosal incision (in a retroflexed position) was performed on the oral side ( $\vee$ Fig. $\mathbf{3 b}$ ). Then, we returned to the individual pockets (water accumulation side first) and resumed the dissection until reaching the previously incised oral side mucosa, thereby creating a tunnel like dissection ( $\vee$ Fig. 3c). Finally, the submucosa between the two pockets was dissected and the lesion was resected en bloc. Triamcinolone $20 \mathrm{mg}$ was injected into the ulcer base to prevent inflammation and subsequent stenosis. Total procedure time was 165 minutes.

The resected specimen resembled a donut-shape resection ( $\triangleright$ Fig.4a,b) with dimensions of $115 \mathrm{~mm} \times 45 \mathrm{~mm}$ ( $\triangleright$ Fig. 4c). Histology showed a tubular adenoma with focal severe atypia. The vertical and horizontal margins of the tumor were negative. The patient did not receive any antibiotics pre- or postprocedure. She did not develop any fever or sepsis and only complained of mild anal pain. The patient was discharged 5 days post-procedure. To prevent stenosis, the patient underwent endoscopic balloon dilation four times using an $18-\mathrm{mm}$ balloon bi-weekly. After these prophylactic interventions, endoscopic stenosis did not occur ( $\triangleright$ Fig.4 d).

\section{Discussion}

The PCM is a tunneling technique, which is generally used for ESD in esophageal neoplasms. However, the rectum has a larger diameter and, in our case, one tunnel was considered insufficient for a rectal circumferential lesion. Therefore, to resolve this, we created two pockets allowing the lesion to be resected en bloc.

An anorectal lesion is a difficult case for endoscopic treatment for three reasons: (1) poor visualization from the narrow anal lumen, (2) presence of hemorrhoidal venous plexus, and (3) anal canal pain [8].

To counter the above difficulties, we used a short ST hood to ensure and maintain a good view. The use of this tapered hood is important for maintaining traction in PCM, allowing dissection even in cases of severe fibrosis. Additionally, it helps to go underneath the tumor allowing for easier dissection compared to a regular non-tapered hood. A report showed three other ad- vantages of the tunneling method, which is theoretically similar to PCM: (1) the dissected part was not mobile; (2) maneuvering with the endoscope was stable and it prevented disorientation; (3) this technique did not require excessive air inflation because insertion of the endoscope into the tunnel itself could help maintain an excellent view [2]. With respect to the ESD knife, we used the ClutchCutter which allowed hemorrhoidal vessels to be caught firmly and dissected safely and, as such, enabled us to stop perioperative hemorrhage from thick vessels without the need for hemostatic forceps.

Previously for anorectal lesions, there was a reported risk of systemic bacteremia from the unique rectal blood supply [8]. However, there are no definitive guidelines on prophylactic antibiotics and we did not administer any antibiotics to this patient.

In terms of the sequence of the pockets, the pocket on the water accumulation side was undertaken first. If we were to resect the opposite side first, the effect of gravity would cause the partially resected specimen to flop and therefore, obstruct both luminal view and instrumentation, making the process more difficult and time consuming.

Previously, severe fibrosis, tumor size more than $50 \mathrm{~mm}$, and poor scope operability were reported to be risk factors for incomplete resection in colorectal ESD [9]. In this case, not only was the size more than $50 \mathrm{~mm}$, but being a circumferential lesion made it difficult and called for an unusual strategy. PCM with two pockets and the combination of the two knives enabled us to resect this large lesion en bloc without perforation or massive procedural hemorrhaging.

Local injection of a corticosteroid in esophageal ESD has proven to prevent subsequent stenosis [10]; however, the efficacy of this has not been reported in colorectal ESD. In our case, we administered a corticosteroid injection and balloon dilation to prevent stenosis, and endoscopic stenosis actually did not occur. Further analysis should be performed to examine the efficacy of local corticosteroid injections. 

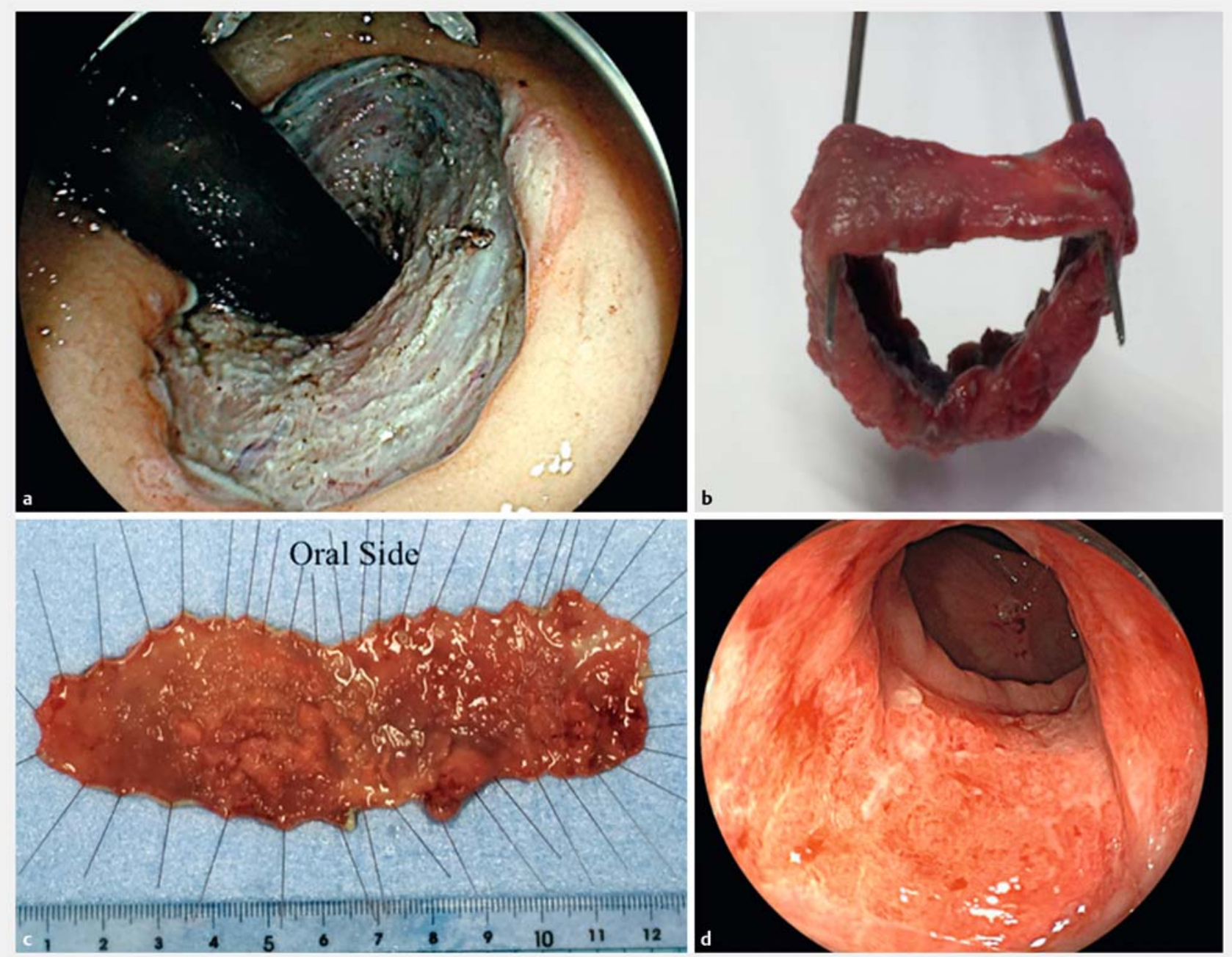

- Fig. 4 a Endoscopic view post-ESD. b En bloc specimen of the full circumferential rectal lesion. c Resected specimen measuring $115 \mathrm{~mm} \times$ $45 \mathrm{~mm}$. d After four prophylactic balloon dilatations, endoscopic stenosis did not occur.

\section{Conclusion}

Circumferential lesions are rare and would be difficult to resect with standard ESD. This case suggests that the PCM with two pockets can be a feasible option in selected cases.

\section{Competing interests}

Yoshito Itoh is affiliated with a donation-funded department from FUJIFILM Medical Co. Ltd. Naohisa Yoshida and Yuji Naito received a research grant from FUJIFILM Medical Co. Ltd. The other authors have no conflicts of interest to declare.

\section{References}

[1] Yamamoto H, Kawata $\mathrm{H}$, Sunada $\mathrm{K}$ et al. Successful en-bloc resection of large superficial tumors in the stomach and colon using sodium hyaluronate and small-caliber-tip transparent hood. Endoscopy 2003; 35: $690-694$
[2] Aslan F, Akpinar Z, Kucuk M et al. Multiple tunneling technique for treatment of rectal circumferential laterally spreading tumor with endoscopic submucosal dissection. VideoGIE 2016; 2: 32-34

[3] Imai K, Hotta K, Yamaguchi Y et al. Submucosal tunneling technique using insulated-tip knife in complete circumferential endoscopic submucosal dissection. Gastrointest Endosc 2016; 84: 742

[4] Hayashi Y, Miura Y, Yamamoto H. Pocket-creation method for the safe, reliable, and efficient endoscopic submucosal dissection of colorectal lateral spreading tumors. Dig Endosc 2015; 27: 534-535

[5] Sano Y, Tanaka S, Kudo SE et al. Narrow-band imaging (NBI) magnifying endoscopic classification of colorectal tumors proposed by the Japan NBI Expert Team. Dig Endosc 2016; 28: 526 - 533

[6] Yoshida N, Hisabe T, Inada Y et al. The ability of a novel blue laser imaging system for the diagnosis of invasion depth of colorectal neoplasms. J Gastroenterol 2014; 49: 73-80

[7] Yoshida N, Naito Y, Murakami T et al. Safety and efficacy of a sameday low-volume $1 \mathrm{~L}$ PEG bowel preparation in colonoscopy for the elderly people and people with renal dysfunction. Dig Dis Sci 2016; 61: 3229-3235

[8] Holt BA, Bassan MS, Sexton A et al. Advanced mucosal neoplasia of the anorectal junction: endoscopic resection technique and outcomes (with videos). Gastrointest Endosc 2014; 79: 119-126 
[9] Takahashi H, Arimura Y, Okahara S et al. A randomized controlled trial of endoscopic steroid injection for prophylaxis of esophageal stenoses after extensive endoscopic submucosal dissection. BMC Gastroenterol 2015; 15: 1
[10] Hayashi N, Tanaka S, Nishiyama S et al. Predictors of incomplete resection and perforation associated with endoscopic submucosal dissection for colorectal tumors. Gastrointest Endosc 2014; 79: 427 435 